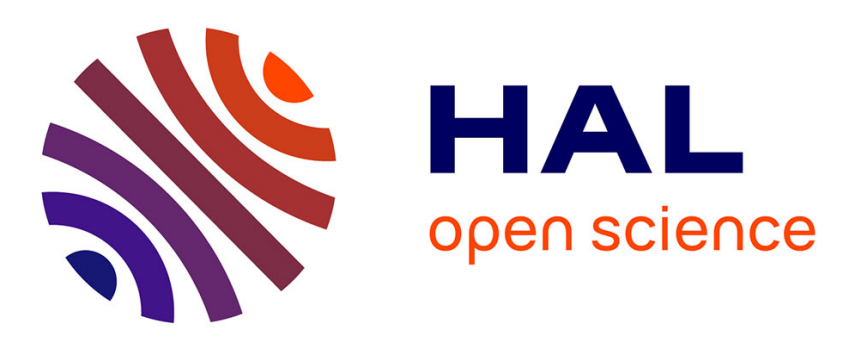

\title{
High gain observer for sensorless induction motor
} Abdou Dib, Mondher Farza, Mohammed M'Saad, Philippe Dorléans, Jean-François Massieu

\section{To cite this version:}

Abdou Dib, Mondher Farza, Mohammed M'Saad, Philippe Dorléans, Jean-François Massieu. High gain observer for sensorless induction motor. The 18th IFAC World Congress on Automatic Control, Aug 2011, Milan, Italy. hal-01060138

\section{HAL Id: hal-01060138 https://hal.science/hal-01060138}

Submitted on 3 Sep 2014

HAL is a multi-disciplinary open access archive for the deposit and dissemination of scientific research documents, whether they are published or not. The documents may come from teaching and research institutions in France or abroad, or from public or private research centers.
L'archive ouverte pluridisciplinaire HAL, est destinée au dépôt et à la diffusion de documents scientifiques de niveau recherche, publiés ou non, émanant des établissements d'enseignement et de recherche français ou étrangers, des laboratoires publics ou privés. 


\title{
High gain observer for sensorless induction motor
}

\author{
A. Dib, M. Farza, M. M'Saad, Ph. Dorléans and J.F. Massieu
}

GREYC UMR 6072 CNRS, Université de Caen, ENSICAEN

6 Boulevard Maréchal Juin, 14050 Caen Cedex, France (e-mail: mondher.farza@unicaen.fr)

\begin{abstract}
The synthesis of a high gain observer for the on-line estimation of the rotor fluxes, the motor speed and the load torque of the induction motor from the current and voltage measurements is presented. The synthesis of the observer is preceded by an observability analysis of the induction motor leading thereby to a sufficient condition. The obtained condition is discussed and it is shown that it is generally satisfied in practice when considering the usual operating conditions of the motor. Of particular interest, an implementation procedure is proposed to deal with the observation singularities. The proposed observer has been validated in simulation using the reference trajectories of a realistic proposed benchmark where the induction motor model remains in an unobservable region and not just go across.
\end{abstract}

Keywords: Nonlinear system, observability, high gain observer, induction motor.

\section{INTRODUCTION}

It is well-known that most control systems for the Induction Motor (IM) require the knowledge of the rotor fluxes as well as that of the angular speed (Leonard [2001]). Since these measurements, in particular those of the fluxes, are not easily accessible, many research efforts have been focused on their estimation in the past few years. Indeed, many alternatives have been studied in order to design observers for IM using Luenberger-like observer (Verghese and Sanders [1988], Martin and Rouchon [2000]), nonlinear observers (Busawon et al. [2001], Lubineau et al. [2000]), sliding mode-based observer (Benchaib et al. [1999]), LMI-based observer (Darengosse et al. [1999]). However, in most of these works, the speed measurement has been supposed to be available and the objective of these works was to provide on-line estimates of the rotor fluxes only. Notice that, in the work of (Ortega et al. [1998]), the authors proposed passivity-based control of IMs which does not make use of the rotor fluxes but the angular speed still be assumed measured. Many other works deal with sensorless control of AC motors drives (Holtz [1996] Tajima and Hori [1993]). Most of these works are based on open loop estimation models which performance reduces as the mechanical speed reduces. Moreover, this performance depends on how precisely the model parameters can be matched to the corresponding parameters in the controlled machine. In order to improve the robustness against parameters mismatch and signal noise, some authors have proposed adaptive observers where the fluxes are estimated using closed loop observers while the angular speed and load torque are treated as time-varying parameters which are adapted through open loop models based on the machine equations (Lascu et al. [2009], Guzinski et al. [2010]). In (Ghanes and Zheng [2009]), the authors proposed a sliding mode like-observer for the on-line estimation of the fluxes as well as the speed and the load torque. The main drawback of the observer lies in the fact that this observer is not easy to implement since its gain involves the signum function and some others design parameters whose values are to be updated when singularities, that are not known a priori, occur. Another observer that provides the estimates of all the states of the IM has also been proposed in (Ghanes et al. [2010]). Again, the main drawback of this observer lies in its implementation which requires the resolution of eighteen differential equations: six equations are associated to the state estimates and the other twelve equations are used to update the observer gain. In (Rossignol et al. [2003]), the authors proposed a high gain observer for the on-line estimation of all the IM states. The fluxes and the motor speed are firstly estimated. Then, the so obtained speed estimate is used as real measurements to estimate the load torque.

In this paper, an observability analysis with an observer synthesis for the IM system is carried out. The considered model accounts for six state variables, namely the two stator currents, the two rotor fluxes, the motor speed and the load torque. The objective of the observability analysis consists in deriving a condition under which all the six states of the motor can be estimated from the current measurements. Then, an appropriate state observer that provides the estimates of the full state is synthesized.

This paper is organized as follows. In the next section, the state space model of the IM which will be the basis of the observability analysis and the observer synthesis is introduced. In section 3, the observability analysis of the IM is carried out and it leads to a sufficient condition ensuring the observability of the IM. Section 4 is devoted to the observer synthesis. In a first step, a class of nonlinear systems including the IM model is introduced and an appropriate high gain observer is synthesized. A full convergence analysis of the observation error is provided. In a second step, the equations of the observer used for the IM are given. Along the observer synthesis procedure, a particular attention has been paid to the implementation aspect. Many simplifications in the observer gain expression have been pointed out and solutions to cope with some singularities in the observer gain are proposed. In section 5 , the performances of the proposed observer are highlighted in simulation by taking into account the unobservability phenomena. Finally, concluding comments are given in section 6 .

\section{PROBLEM FORMULATION}

The electrical behavior of an IM can be described in the $(\alpha, \beta)$ coordinates system in stationary reference frame fixed with the stator. Under the assumption that the dynamics of the load torque is bounded, the IM model is given by the following set of state variables equations (Leonard [2001]):

$$
\left\{\begin{array}{l}
\dot{i}=N F(\omega) \psi-\gamma i+\frac{1}{\sigma L_{s}} u \\
\dot{\psi}=-F(\omega) \psi+\frac{M}{T_{r}} i \\
\dot{\omega}=\frac{p M}{J L_{r}} i^{T} J_{2} \psi-\frac{1}{J} T_{L} \\
\dot{T}_{L}=\varepsilon(t)
\end{array}\right.
$$


where $i=\left(i_{1}, i_{2}\right)^{T}, \psi=\left(\psi_{1}, \psi_{2}\right)^{T}, u=\left(u_{1}, u_{2}\right)^{T}$ are respectively the stator currents, the rotor fluxes and the voltages; $\omega$ and $T_{L}$ respectively denote the motor speed and the load torque; $\varepsilon$ is an unknown bounded function; $F(\omega)=\frac{1}{T_{r}} I_{2}-p \omega J_{2}, I_{2}$ is the $2 \times 2$ identity matrix and $J_{2}=\left(\begin{array}{cc}0 & -1 \\ 1 & 0\end{array}\right) ; J$ is the motor moment of inertia; $p$ is the number of pairs of poles. The parameters $T_{r}, \sigma$, $N$ and $\gamma$ are defined as follows:

$T_{r}=\frac{L_{r}}{R_{r}}, \sigma=1-\frac{M^{2}}{L_{s} L_{r}}, N=\frac{M}{\sigma L_{s} L_{r}}, \gamma=\frac{R_{s}}{\sigma L_{s}}+\frac{R_{r} M^{2}}{\sigma L_{s} L_{r}^{2}}$ where $R_{s}$, $R_{r}$ are stator (resp. rotor) per-phase resistances, $L_{s}, L_{r}$ are stator (resp. rotor) per-phase inductances and $M$ is the mutual inductance.

Notice that the time derivative of the load torque is described by an unknown bounded function. The objective is to determine under what conditions all the state of the motor, $i, \psi, \omega$ and $T_{L}$ can be determined from the output and input measurements, namely the current and the voltage measurements $i$ and $u$, respectively. This leads us to study the observability of system (1) by considering $y=i$ as output. For clarity purposes, one introduces the following notations:

$$
\begin{aligned}
& x=\left(\begin{array}{l}
x^{1} \\
x^{2} \\
x^{3}
\end{array}\right) \text { with } x^{1}=\left(\begin{array}{l}
x_{1}^{1} \\
x_{2}^{1}
\end{array}\right), x^{2}=\left(\begin{array}{c}
x_{1}^{2} \\
x_{2}^{2}
\end{array}\right), x^{3}=\left(\begin{array}{c}
x_{1}^{3} \\
x_{2}^{3}
\end{array}\right) \\
& x_{1}^{1}=i_{1}, x_{2}^{1}=i_{2}, x_{1}^{2}=\psi_{1}, x_{2}^{2}=\psi_{2}, x_{1}^{3}=\omega, x_{2}^{3}=T_{L}
\end{aligned}
$$

In the sequel, the notation $I_{k}$ and $0_{k}$ shall be used to denote the $k \times k$ identity matrix and the $k \times k$ null matrix, respectively. The rectangular $k \times m$ null matrix shall be denoted by $0_{k \times m}$.

System (1) can then be written under the following condensed form:

$$
\left\{\begin{array}{l}
\dot{x}=f(u, x)+B_{1} \varepsilon(t) \\
y=x^{1}
\end{array}\right.
$$

where

$$
f(u, x)=\left(\begin{array}{c}
N F\left(x_{1}^{3}\right) x^{2}-\gamma x^{1}+\frac{1}{\sigma L_{s}} u \\
-F\left(x_{1}^{3}\right) x^{2}+\frac{M}{T_{r}} x^{1} \\
\frac{p M}{J L_{r}} x^{1 T} J_{2} x^{2}-\frac{1}{J} x_{2}^{3} \\
0
\end{array}\right), B_{1}^{T}=\left[\begin{array}{ll}
0_{1 \times 5} & 1
\end{array}\right]
$$

\section{OBSERVABILITY ANALYSIS OF THE IM MODEL}

In this section, one shall introduce a classical state transformation that puts system (1) under a known observable canonical form. Then, one shall look for a sufficient condition under which the considered state transformation is a diffeomorphism. In particular, this analysis shall give rise to a condition under which the jacobian of the considered transformation is of full rank almost everywhere. Please notice that the observability of the induction model has been fully treated in (Wit et al. [2000]) and (Ibarra-Rojas et al. [2004]). But no observer has been proposed in either of these papers. In (Wit et al. [2000]), the authors give a sufficient condition ensuring the observability of the IM. A nice characterization of the motor (un)observability is provided in (Ibarra-Rojas et al. [2004]) where it was shown that the indistinguishable dynamics of the IMs are governed by an Algebraic Differential Equations system. In what follows, an observability analysis is fully carried out with view to observer synthesis. Most of the developments detailed through this analysis are used when synthesizing the observer.

Now, let us consider the following change of variables

$$
\Phi: \mathbb{R}^{6} \rightarrow \mathbb{R}^{6}, x \mapsto z=\left(\begin{array}{c}
z^{1} \\
z^{2} \\
z^{3}
\end{array}\right)=\Phi(x)=\left(\begin{array}{c}
\Phi^{1}(x) \\
\Phi^{2}(x) \\
\Phi^{3}(x)
\end{array}\right)
$$

where the $\Phi^{k}$ 's, $k=1,2,3$, are defined as follows:

$$
\left\{\begin{aligned}
z^{1}=\Phi^{1}(x)= & x^{1} \\
z^{2}=\Phi^{2}(x)= & N F\left(x_{1}^{3}\right) x^{2} \\
= & N\left(\frac{1}{T_{r}} I_{2}-p x_{1}^{3} J_{2}\right) x^{2} \\
z^{3}=\Phi^{3}(x)= & -p N J_{2}\left(\dot{x}_{1}^{3} x^{2}+x_{1}^{3} \dot{x}^{2}\right) \\
= & -p N J_{2}\left(\frac{1}{J}\left(\frac{p M}{L_{r}} x^{1 T} J_{2} x^{2}-x_{2}^{3}\right) x^{2}\right. \\
& \left.+x_{1}^{3}\left(-F\left(x_{1}^{3}\right) x^{2}+\frac{M}{T_{r}} x^{1}\right)\right)
\end{aligned}\right.
$$

with

$$
F\left(x_{1}^{3}\right)=\frac{1}{T_{r}} I_{2}-p x_{1}^{3} J_{2}=\left[\begin{array}{cc}
\frac{1}{T_{r}} & p x_{1}^{3} \\
-p x_{1}^{3} & \frac{1}{T_{r}}
\end{array}\right]
$$

One can show that the above state transformation puts system (1) under the following form:

$$
\left\{\begin{array}{l}
\dot{z}^{1}=z^{2}+\varphi^{1}\left(u, z^{1}\right) \\
\dot{z}^{2}=z^{3}+\varphi^{2}\left(z^{1}, z^{2}\right) \\
\dot{z}^{3}=\varphi^{3}(z)+b(z) \varepsilon(t) \\
y=C z=z^{1}
\end{array}\right.
$$

with $z=\left(\begin{array}{c}z^{1} \\ z^{2} \\ z^{3}\end{array}\right) ; z^{k}=\left(\begin{array}{c}z_{1}^{k} \\ z_{2}^{k}\end{array}\right)$ for $k=1,2,3 ; \varepsilon(t)$ is given by system (1) and the nonlinear functions $b$ and $\varphi^{k} \in \mathbb{R}^{2}, k=1,2,3$ are defined as follows:

$$
\left\{\begin{aligned}
\varphi^{1}\left(u, z^{1}\right)= & -\gamma z^{1}+\frac{1}{\sigma L_{s}} u \\
\varphi^{2}\left(z^{1}, z^{2}\right)= & \frac{1}{T_{r}}\left(-z^{2}+\frac{M N}{T_{r}} z^{1}\right) \\
\varphi^{3}(z) \triangleq & \frac{\partial \Phi^{3}}{\partial x^{1}}(u, x) \dot{x}^{1}+\frac{\partial \Phi^{3}}{\partial x^{2}}(u, x) \dot{x}^{2} \\
& +\frac{\partial \Phi^{3}}{\partial x_{1}^{3}}(u, x) \dot{x}_{1}^{3} \\
b(z) \triangleq & \frac{\partial \Phi^{3}}{\partial x_{2}^{3}}(u, x)
\end{aligned}\right.
$$

Please notice that, system (7) is observable for any input $u$. As a result, system (1) shall be observable in the rank sense on $\mathbb{R}^{6}$ as soon as the transformation $\Phi$ exists and is regular almost everywhere (Hermann and Krener [1977]). Indeed one shall exhibit a sufficient condition under which the jacobian of this transformation is of full rank almost everywhere.

Let $J_{\Phi}$ be the jacobian of $\Phi$. According to (5), one has

$$
J_{\Phi}(x)=\left[\begin{array}{ccc}
I_{2} & 0_{2} & 0_{2} \\
0_{2} & \frac{\partial \Phi^{2}}{\partial x^{2}}(x) & \frac{\partial \Phi^{2}}{\partial x^{3}}(x) \\
\frac{\partial \Phi^{3}}{\partial x^{1}}(x) & \frac{\partial \Phi^{3}}{\partial x^{2}}(x) & \frac{\partial \Phi^{3}}{\partial x^{3}}(x)
\end{array}\right]
$$

It is clear that the matrix $J_{\Phi}(x)$ is of full rank if and only if the following square matrix is also of full rank:

$$
G_{\Phi}(x)=\left[\begin{array}{ll}
\frac{\partial \Phi^{2}}{\partial x^{2}}(x) & \frac{\partial \Phi^{2}}{\partial x^{3}}(x) \\
\frac{\partial \Phi^{3}}{\partial x^{2}}(x) & \frac{\partial \Phi^{3}}{\partial x^{3}}(x)
\end{array}\right] \triangleq\left[\begin{array}{ll}
G_{1}(x) & G_{2}(x) \\
G_{3}(x) & G_{4}(x)
\end{array}\right]
$$

In what following, one shall focus on the matrix $G_{\Phi}$ in order to exhibit a sufficient condition under which this matrix, or equivalently $J_{\Phi}$, is of full rank almost everywhere. To this end, one shall compute each entry of $G_{\Phi}(x)$. Again, according to (5), one has: 


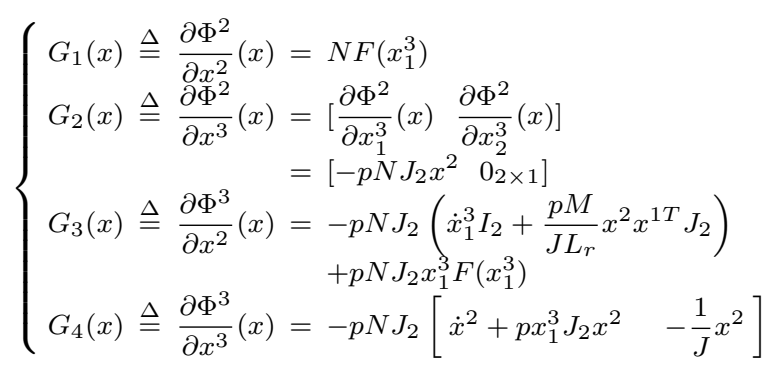

It is clear from (6) that $G_{1}(x)$ is a square invertible matrix for all $x \in \mathbb{R}^{6}$. As a result, the matrix $G_{\Phi}(x)$ can be factorized as follows:

$$
\begin{aligned}
G_{\Phi}(x) & =L(x) U(x) \\
L(x) & =\left[\begin{array}{cc}
I_{2} & 0_{2} \\
G_{3}(x) G_{1}^{-1}(x) & G_{4}(x)-G_{3}(x) G_{1}^{-1}(x) G_{2}(x)
\end{array}\right] \\
U(x) & =\left[\begin{array}{cc}
G_{1}(x) & G_{2}(x) \\
0_{2} & I_{2}
\end{array}\right]
\end{aligned}
$$

From the structures of $L$ and $U$, one can deduce that the matrix $G_{\Phi}(x)$ is of full rank if and only if the block triangular lower matrix $L(x)$ is also of full rank. According to its triangular structure, the full rank condition of $L(x)$ is obtained as soon as the matrix

$$
L_{2}(x) \triangleq G_{4}(x)-G_{3}(x) G_{1}^{-1}(x) G_{2}(x)
$$

is of full rank. In order to check the full rank condition of the matrix $L_{2}(x)$, we shall check the linear dependence of its two columns. Indeed, set $L_{2}(x)=\left[\begin{array}{ll}L_{21}(x) & L_{22}(x)\end{array}\right]$ and let us firstly derive the expression of $L_{21}$ and $L_{22}$. Using (10), one gets:

$$
\begin{aligned}
L_{21}(x)= & -p N J_{2}\left(\dot{x}^{2}+\left(\dot{x}_{1}^{3} F_{1}^{-1}\left(x_{1}^{3}\right)\right.\right. \\
& \left.\left.+\frac{p M}{J L_{r}} x^{2} x^{1 T} J_{2} F_{1}^{-1}\left(x_{1}^{3}\right)\right) p J_{2} x^{2}\right) \\
\triangleq & -p N J_{2} M(x) \\
L_{22}(x)= & p N J_{2} \frac{1}{J} x^{2}
\end{aligned}
$$

Now, the columns $L_{21}(x)$ and $L_{22}(x)$ are linearly dependent if and only if $M(x)$ and $x^{2}$ are so. This is also equivalent to $x^{2 T} J_{2} M(x)=0$. This condition can be expressed under the following more explicit form:

$$
x^{2 T} J_{2} M(x)=x^{2 T} J_{2} \dot{x}^{2}-\frac{p}{T_{r}} \frac{\dot{x}_{1}^{3}}{\left(\frac{1}{T_{r}}\right)^{2}+\left(p x_{1}^{3}\right)^{2}} x^{2 T} x^{2}
$$

To summarize, the considered transformation has a full rank (i.e. system (1) is observable) in each $x$ as soon as

$$
x^{2 T} J_{2} \dot{x}^{2}-\frac{p}{T_{r}} \frac{\dot{x}_{1}^{3}}{\left(\frac{1}{T_{r}}\right)^{2}+\left(p x_{1}^{3}\right)^{2}} x^{2 T} x^{2} \neq 0 \text { a.e. }
$$

Condition (15) can be explained by using the original motor variables as follows:

$$
\psi^{T} J_{2} \dot{\psi}-\frac{p}{T_{r}} \frac{\dot{\omega}}{\left(\frac{1}{T_{r}}\right)^{2}+(p \omega)^{2}} \psi^{T} \psi \neq 0 \text { a.e. }
$$

Condition (16) can be expressed in different equivalent forms. Indeed, one shall exhibit such equivalent forms and comment them. Let us define the flux angle rotor as follows: $\xi=\arctan \frac{\psi_{2}}{\psi_{1}}$. From the motor model (1), one has $\dot{\xi}=-\frac{\psi^{T} J_{2} \dot{\psi}}{\psi^{T} \psi}$ and by excluding the uninteresting case of $\psi=0$, condition (16) can be explained as follows:

$$
\dot{\xi}(t) \neq \frac{p}{T_{r}} \frac{\dot{\omega}(t)}{\left(\frac{1}{T_{r}}\right)^{2}+(p \omega(t))^{2}} \text { a.e. }
$$

or equivalently by taking the integral of both sides

$$
\left(\arctan \frac{\psi_{2}(t)}{\psi_{1}(t)}-\arctan \left(p T_{r} \omega(t)\right)\right) \quad \text { is not constant a.e. }
$$

Notice that conditions (16) and (17) are similar to those given in (Wit et al. [2000]).

\section{OBSERVER DESIGN}

The aim of this section is to propose a state observer for system (1). Such observer has to provide on-line estimation of $i, \psi, \omega$ and $T_{L}$ from the sole measurements of the stator currents $i$ and the voltages $u$ without making use of the time derivatives of these measurements since they are unavailable. To this end, one shall focus on a class of systems that includes the motor state model with a view of observer synthesis. Then the proposed result shall be applied to the IM model in order to derive a state observer that provides full state estimates.

\subsection{Observer design for a class of nonlinear systems}

Consider the following dynamical system:

$$
\left\{\begin{array}{l}
\dot{x}(t)=f(u, x)+B_{1} \varepsilon(t) \\
y(t)=C x(t)=x^{1}(t)
\end{array}\right.
$$

where the state $x=\left(\begin{array}{c}x^{1} \\ x^{2} \\ \vdots \\ x^{q}\end{array}\right) \in \mathbb{R}^{n}, x^{k} \in \mathbb{R}^{p}, k=1, \ldots, q$;

$f(u, x)=\left(\begin{array}{c}f^{1}(u, x) \\ f^{2}(u, x) \\ \vdots \\ f^{q-1}(u, x) \\ f^{q}(u, x)\end{array}\right)$; the input $u \in \Omega$ a compact subset of

$\mathbb{R}^{s}$; the output $y \in \mathbb{R}^{p}$; the matrix $C$ is $p \times n$ and is defined as follows:

$$
C=\left[I_{p}, 0_{p}, \ldots, 0_{p}\right]
$$

Finally, $B_{1}=[0 \ldots 0,1]^{T} \in \mathbb{R}^{n}$ and $\varepsilon(t) \in \mathbb{R}$ is an unknown bounded function, i.e. $\exists \delta>0 ; \forall t \geq 0:\|\varepsilon(t)\| \leq \delta$.

One assumes that there exists a Lipschitzian diffeomorphism $\Phi$ : $\mathbb{R}^{n} \longrightarrow \mathbb{R}^{n}, x \mapsto z=\Phi(x)$ that puts system (19) under the following form:

$$
\left\{\begin{array}{l}
\dot{z}=A z+\varphi(u, z)+B b(z) \varepsilon(t) \\
y=C z=z^{1}
\end{array}\right.
$$

where the state $z=\left(\begin{array}{c}z^{1} \\ z^{2} \\ \vdots \\ z^{q}\end{array}\right) \in \mathbb{R}^{n}$ with $z^{k} \in \mathbb{R}^{p}, k=$ $1, \ldots, q$ (i.e. $n=p q), b(z)$ is $p \times p$ bounded matrix, the function $\varphi(u, z)$ has a triangular structure with respect to $z$, i.e. $\varphi(u, z)=$ $\left(\begin{array}{c}\varphi^{1}\left(u, z^{1}\right) \\ \varphi^{2}\left(u, z^{1}, z^{2}\right) \\ \vdots \\ \varphi^{q-1}\left(u, z^{1}, \ldots, z^{q-1}\right) \\ \varphi^{q}(u, z)\end{array}\right) \in \mathbb{R}^{n}$; the matrix $A$ is the following antishift block matrix 


$$
A=\left[\begin{array}{cc}
0 & I_{(q-1) p} \\
0 & 0
\end{array}\right]
$$

and finally the matrix $B$ is defined as follows:

$$
B^{T}=\left[0_{p}, \ldots, 0_{p}, I_{p}\right]
$$

Notice that, if an observer is synthesized for system (21), then its implementation in the original coordinates requires to compute the inverse of the jacobian transformation. This could be time consuming and could constitutes an obstacle for the real time implementation of such observer. According to this, one shall synthesize an observer for system (21) such that its implementation in the original coordinates does not systematically require the computation of all the entries of the jacobian inverse. To this end, one introduces the following decomposition of the jacobian inverse:

$$
\left(\frac{\partial \Phi}{\partial x}(\hat{x})\right)^{-1}=\Lambda_{U}(\hat{x})+\Lambda_{L}(\hat{x})
$$

where $\Lambda_{U}(\hat{x})$ is an upper triangular block matrix and $\Lambda_{L}(\hat{x})$ is a lower triangular block matrix satisfying the following structure condition:

$$
\Gamma(\hat{x}) \triangleq\left(\frac{\partial \Phi}{\partial x}(\hat{x})\right) \Lambda_{L}(\hat{x})
$$

(25) is a lower triangular matrix with zeros on the main diagonal.

Before giving the equations of the candidate observer, one introduces the following definitions and notations:

- Let $\Delta_{\theta}$ be the (block) diagonal matrix defined as follows:

$$
\Delta_{\theta}=\operatorname{diag}\left(I_{p}, \frac{1}{\theta} I_{p}, \ldots, \frac{1}{\theta^{q-1}} I_{p}\right)
$$

where $\theta>0$ is a design parameter.

- Let $K$ be a $p q \times p$ rectangular matrix such that $A-K C$ is Hurwitz. The choice of such matrix is of course always possible since the pair $(A, C)$ is observable.

The design of the proposed observer requires the following technica assumption which is usually used when designing high gain observers:

(H) The function $\varphi$ is globally Lipschitz with respect to $z$ uniformly in $u$.

Now, consider the following dynamical system:

$$
\dot{\hat{x}}=f(u, \hat{x})-\theta \Lambda_{U}(\hat{x}) \Delta_{\theta}^{-1} K(C \hat{x}-y)
$$

where $\hat{x}=\left(\begin{array}{c}\hat{x}^{1} \\ \hat{x}^{2} \\ \vdots \\ \hat{x}^{q}\end{array}\right) \in \mathbb{R}^{n}$ with $\hat{x}^{k} \in \mathbb{R}^{p} ; u$ and $y$ are respectively the input and output of system (19).

One states the following:

Theorem Under assumption (H), system (27) is an observer for system (19) with the following property: in the absence of uncertainties (i.e. $\varepsilon=\beta=0$ ), the observation error converges exponentially to zero. In the case where $\varepsilon(t) \neq 0$, the observation error is ultimately bounded and the corresponding ultimate bound can be made as small as desired by choosing values of $\theta$ high enough.

Proof of the theorem: the proof of the theorem is similar to that given in (Farza et al. [2004]) and is omitted due to the lack of place.

\subsection{Observer design for the IM}

In this section, one assumes that the state transformation defined by (4)-(5) is regular almost everywhere. Such condition is satisfied by assuming that one from the two equivalent conditions given by (17) or (18) is satisfied. Indeed, this shall be assumed. According to (8) and (9), the jacobian $J_{\Phi}$ of the state transformation can be written as follows:

$$
J_{\Phi}(x)=\left[\begin{array}{cc}
I_{2} & 0_{2 \times 4} \\
H_{\Phi}(x) & G_{\Phi}(x)
\end{array}\right]
$$

where $G_{\Phi}(x)$ is given by $(9)$ and $H_{\Phi}(x)=\left[\begin{array}{c}0_{2 \times 2} \\ \frac{\partial \Phi^{3}}{\partial x^{1}}(u, x)\end{array}\right]$. It is clear that under condition (17), $J_{\Phi}(x)$ is invertible almost everywhere. At this step, one assumes that $J_{\Phi}(x)$ is invertible everywhere. Then, one shall show how to deal with singular points, i.e. isolated points where the jacobian is singular. According to (8), the inverse of $J_{\Phi}(x)$ is:

$$
J_{\Phi}^{-1}(x)=\left[\begin{array}{cc}
I_{2} & 0_{2 \times 4} \\
-G_{\Phi}^{-1}(x) H_{\Phi}(x) & G_{\Phi}^{-1}(x)
\end{array}\right]
$$

The matrix $J_{\Phi}^{-1}(x)$ can be partitioned as follows:

$$
\begin{aligned}
J_{\Phi}^{-1}(x) & =\Lambda_{L}(x)+\Lambda_{U}(x) \\
\text { with: } \Lambda_{L}(x) & =\left[\begin{array}{cc}
0_{2} & 0_{2 \times 4} \\
-G_{\Phi}^{-1}(x) H_{\Phi}(x) & 0_{4 \times 4}
\end{array}\right] \\
\Lambda_{U}(x) & =\left[\begin{array}{cc}
I_{2} & 0_{2 \times 4} \\
0_{4 \times 2} & G_{\Phi}^{-1}(x)
\end{array}\right]
\end{aligned}
$$

One clearly has

$$
J_{\Phi}(x) \Lambda_{L}(x)=\left[\begin{array}{cc}
0_{2} & 0_{2 \times 4} \\
-G_{\Phi}(x) G_{\Phi}^{-1}(x) H_{\Phi}(x) & 0_{4 \times 4}
\end{array}\right]
$$

As a result, $\Lambda_{L}(x)$ given by (30) satisfies condition (25) and the following dynamical system is an observer for the IM represented by its model (3):

$$
\dot{\hat{x}}=f(u, \hat{x})-\theta \Lambda_{U}(\hat{x}) \Delta_{\theta}^{-1} K\left(C \hat{x}-x^{1}\right)
$$

where $\Lambda_{U}(\hat{x})$ is given by (30) and $K$ is a matrix such that $A-K C$ is Hurwitz where $A, C$ and $\Delta_{\theta}$ are respectively given by (22), (20) and (26) with $p=2$.

Some problems may occur if no precaution are taken when implementing observer (32). Indeed, the transformation jacobian (or equivalently the matrix $G_{\Phi}(\hat{x})$ is assumed to be regular almost everywhere. This condition does not prevent it from being singular at some isolated points where the inverse of the matrix (or equivalently $\left.\Lambda_{U}(\hat{x})\right)$ cannot be computed. In order to prevent this problem, one shall implement observer (32) by judiciously approximating the matrix $\Lambda_{U}(\hat{x})$. One proposes in what follows to describe and discuss the approximation of $\Lambda_{U}(\hat{x})$.

In fact, according to the structure of $J_{\Phi}(\hat{x})$, the approximation of $\Lambda_{U}(\hat{x})$ is reduced to look for an approximation of $G_{\Phi}(\hat{x})$. To this end, let us consider the factorization of $G_{\Phi}(\hat{x})$ under form (11). It is clear from the structures of $L(\hat{x})$ and $U(\hat{x})$ and from the fact that $U(\hat{x})$ is invertible everywhere that the matrices $G_{\Phi}(\hat{x})$ and $L_{2}(\hat{x})$ given by (12) have the same singular points. As a result, it suffices to look for an approximation for the inverse of $L_{2}(\hat{x})$ in order to approximate $G_{\Phi}(\hat{x})$. The inverse of $L_{2}(\hat{x})$ shall be approximated by a generalized inverse, denoted $L_{2}^{+}(\hat{x})$, that can be expressed as follows:

$$
L_{2}^{+}(\hat{x})=\left(L_{2}^{T}(\hat{x}) L_{2}(\hat{x})+\delta I_{2}\right)^{-1} L_{2}^{T}(\hat{x})
$$


where $\delta>0$ is chosen arbitrarily small. Equation (33) can be written in a more explicit form useful for real time implementation. To derive such expression and for notation convenience, one shall rename the entries of the matrix $L_{2}(\hat{x})$ given by (13) as follows:

$$
L_{2}(\hat{x})=\left[\begin{array}{ll}
l_{1}(\hat{x}) & l_{2}(\hat{x}) \\
l_{3}(\hat{x}) & l_{4}(\hat{x})
\end{array}\right]
$$

With $L_{2}(\hat{x})$ described by (34), equation (33) specializes as follows:

$$
L_{2}^{+}(\hat{x})=\frac{\operatorname{det}\left(L_{2}(\hat{x})\right) \operatorname{adj} L_{2}(\hat{x})+\delta L_{2}(\hat{x})}{\left(\operatorname{det}\left(L_{2}(\hat{x})\right)\right)^{2}+\delta \sum_{i=1}^{4} l_{i}^{2}(\hat{x})+\delta^{2}}
$$

where $\operatorname{det}\left(L_{2}(\hat{x})\right)=l_{1}(\hat{x}) l_{4}(\hat{x})-l_{2}(\hat{x}) l_{3}(\hat{x})$ is the determinant of $L_{2}(\hat{x})$ and $\operatorname{adj} L_{2}(\hat{x})=\left[\begin{array}{cc}l_{4}(\hat{x}) & -l_{2}(\hat{x}) \\ -l_{3}(\hat{x}) & l_{1}(\hat{x})\end{array}\right]$ is the adjoint of matrix $L_{2}(\hat{x})$. From equation $(35)$, one notices that:

- In all the points $\hat{x}$ where the matrix $L_{2}(\hat{x})$ is invertible, or more precisely where $\operatorname{det}\left(L_{2}(\hat{x})\right)>>\delta, \delta$ being an arbitrarily small positive number, one has $L_{2}^{+}(\hat{x}) \# \frac{\operatorname{adj} L_{2}(\hat{x})}{\operatorname{det}\left(L_{2}(\hat{x})\right)}=L_{2}^{-1}(\hat{x})$.

- When the matrix $L_{2}(\hat{x})$ is singular, $L_{2}^{+}(\hat{x})$ is a normalized version of $L_{2}(\hat{x})$.

According to the above developments and using the decomposition of $G_{\Phi}(\hat{x})$ under form (11), the approximation of the inverse of $G_{\Phi}(\hat{x})$, denoted by $G_{\Phi}^{+}(\hat{x})$, can be written as follows:

$$
\begin{aligned}
& G_{\Phi}^{+}(\hat{x})=U^{-1}(\hat{x}) L^{+}(\hat{x}) \\
& =\left[\begin{array}{cc}
G_{1}^{-1}(\hat{x}) & -G_{1}^{-1}(\hat{x}) G_{2}(\hat{x}) \\
0_{2} & I_{2}
\end{array}\right] \times \\
& {\left[\begin{array}{cc}
I_{2} & 0_{2} \\
-L_{2}^{+}(\hat{x}) G_{3}(\hat{x}) G_{1}^{-1}(x) & L_{2}^{+}(\hat{x})
\end{array}\right]} \\
& \triangleq\left[\begin{array}{ll}
\Upsilon_{1} & \Upsilon_{2} \\
\Upsilon_{3} & \Upsilon_{4}
\end{array}\right]
\end{aligned}
$$

where

$$
\begin{aligned}
& \Upsilon_{1}=G_{1}^{-1}(\hat{x})+G_{1}^{-1}(\hat{x}) G_{2}(\hat{x}) L_{2}^{+}(\hat{x}) G_{3}(x) G_{1}^{-1}(x) \\
& \Upsilon_{2}=-G_{1}^{-1}(\hat{x}) G_{2}(\hat{x}) L_{2}^{+}(\hat{x}) \\
& \Upsilon_{3}=-L_{2}^{+}(\hat{x}) G_{3}(\hat{x}) G_{1}^{-1}(x) \text { and } \Upsilon_{4}=L_{2}^{+}(\hat{x})
\end{aligned}
$$

Using (37), the matrix $\Lambda_{U}(\hat{x})$ intervening in the equation of observer (32) can then be computed as follows:

$$
\Lambda_{U}(\hat{x})=\left[\begin{array}{cc}
I_{2} & 0_{2 \times 4} \\
04 \times 2 & G_{\Phi}^{+}(\hat{x})
\end{array}\right]
$$

To summarize, the observer (27) for the IM specializes as follows:

$$
\left\{\begin{aligned}
\dot{\hat{i}} \quad & N F(\hat{\omega}) \hat{\psi}-\gamma \hat{i}+\frac{1}{\sigma L_{s}} u-k_{1} \theta \tilde{i} \\
\dot{\hat{\psi}}= & -F(\hat{\omega}) \hat{\psi}+\frac{M}{T_{r}} \hat{i}-\theta^{2}\left(k _ { 2 } \left(G_{1}^{-1}(\hat{x})\right.\right. \\
& \left.+G_{1}^{-1}(\hat{x}) G_{2}(\hat{x}) L_{2}^{+}(\hat{x}) G_{3}(x) G_{1}^{-1}(x)\right) \\
& \left.-k_{3} \theta G_{1}^{-1}(\hat{x}) G_{2}(\hat{x}) L_{2}^{+}(\hat{x})\right) \tilde{i} \\
\left(\begin{array}{c}
\dot{\hat{\omega}} \\
\dot{\hat{T}}
\end{array}\right)= & \left(\begin{array}{c}
p M \\
J L_{r}
\end{array} \hat{i}^{T} J_{2} \hat{\psi}-\frac{1}{J} \hat{T}_{L}\right. \\
& -\theta^{2}\left(k_{2} L_{2}^{+}(\hat{x}) G_{3}(\hat{x}) G_{1}^{-1}(x)-k_{3} \theta L_{2}^{+}(\hat{x})\right) \tilde{i}
\end{aligned}\right.
$$

where $\tilde{i}=\hat{i}-i, i$ being the available current measurements. Notice that the gain matrix $K$ in observer (38) has been chosen as: $K^{T}=$ $\left(\begin{array}{lll}k_{1} I_{2} & k_{2} I_{2} & k_{3} I_{2}\end{array}\right)$

\section{SIMULATION RESULTS}

The performances of the proposed observer shall be illustrated in simulation in this section. To this end, a dedicated benchmark has been defined. It particularly allows to test the observer performances in open loop around the unobservability region defined in the above section. The dedicated benchmark is defined by specifying the following operating conditions during simulation which started at 0s and ended at 11s (Ghanes and Zheng [2009]):

- Low speed with nominal load from 1s to 3s.

- High speed with nominal load from $4 \mathrm{~s}$ to $6 \mathrm{~s}$.

- Very low speed (zero frequency, the IM is unobservable) with nominal load from $7 \mathrm{~s}$ to $9 \mathrm{~s}$.

- The norm of the fluxes vector, $\|\psi(t)\|$, is constant and equal to 0.6 $\mathrm{Wb}$ along the experiment i.e. from $0 \mathrm{~s}$ to $11 \mathrm{~s}$.

In order to obtain the desired trajectory of the speed, $\bar{\omega}$, a signal satisfying the above requirements, namely $\omega_{i n}$ has been considered as the input of a filter with a pole of order three placed in (-30). The three states of the filter are then the motor desired speed, $\bar{z}_{1}$ and its first and second time derivatives that shall be denoted by $\dot{\bar{z}}_{1}$ and $\ddot{\bar{z}}_{1}$, respectively. Similarly, the desired trajectory of the load torque, $\bar{T}_{L}$ is obtained as the output of a filter with a double pole in (-30) to which the piecewise constant signal, $T_{L i n}$, satisfying the above requirements, is applied. The two states of the second filter are then the applied load torque, $\bar{T}_{L}$ and its first time derivative that shall be denoted by $\dot{\bar{T}}_{L}$.

The voltage, $\bar{u}$ allowing the obtaining of the above desired state trajectories is computed as in (Hajji et al. [2008]) where the authors proposed a high gain control law for the induction model. One emphasizes that the model is not controlled during simulation but is in an open loop. The above expressions of the inputs only allow to obtain the desired trajectories. The parameters values used to simulate model (1) and observer (38) correspond to a $1.5 k \mathrm{~W}$ IM. These are:

$$
\begin{gathered}
p=2 ; \quad L s=0.105 H ; \quad M=0.094 H ; J=0.0077 K_{g} \cdot m^{2} \\
L r=0.094 H ; R s=1.47 \Omega ; R r=0.79 \Omega
\end{gathered}
$$

The initial values for the non measured states, $\hat{\psi}(0), \hat{\omega}(0)$ and $\hat{T}_{L}(0)$ are arbitrarily set to zero which give rise to a non zero initial observation error. Notice that, and as stated above, the reference trajectories have been chosen such that the IM becomes unobservable between $7 \mathrm{~s}$ and $9 \mathrm{~s}$. In order to put forward the performance of the observer, the inputs of the IM model have been corrupted by an additive constant between $8 \mathrm{~s}$ and $8.5 \mathrm{~s}$ i.e. when the IM is unobservable. This perturbation is unknown by the observer. Estimation results are reported in Figure 1 that clearly shows the good performance of the observer in providing accurate estimate even in the case where the unobservability region is met. The obtained results suggest the following comments:

- At the beginning of the simulation, the IM model is observable and the estimates provided by the observer quickly converge to the unknown trajectories in spite of the erroneous state observer initial conditions.

- At the time $7 s$, the IM model becomes unobservable but each state of the observer coincide at that moment with the corresponding state of the IM model. So, there is no error on the initial conditions at the entry of the unobservability region and the provided estimates still coincide with the true trajectories.

- Between $8 s$ and $8.5 s$ the IM model has been corrupted by a disturbance which is unknown by the observer. Since the IM model still be unobservable, the provided estimates deviated from the real values. But as soon as the IM model becomes observable (at 9s), these estimates quickly converge to the true trajectories. 

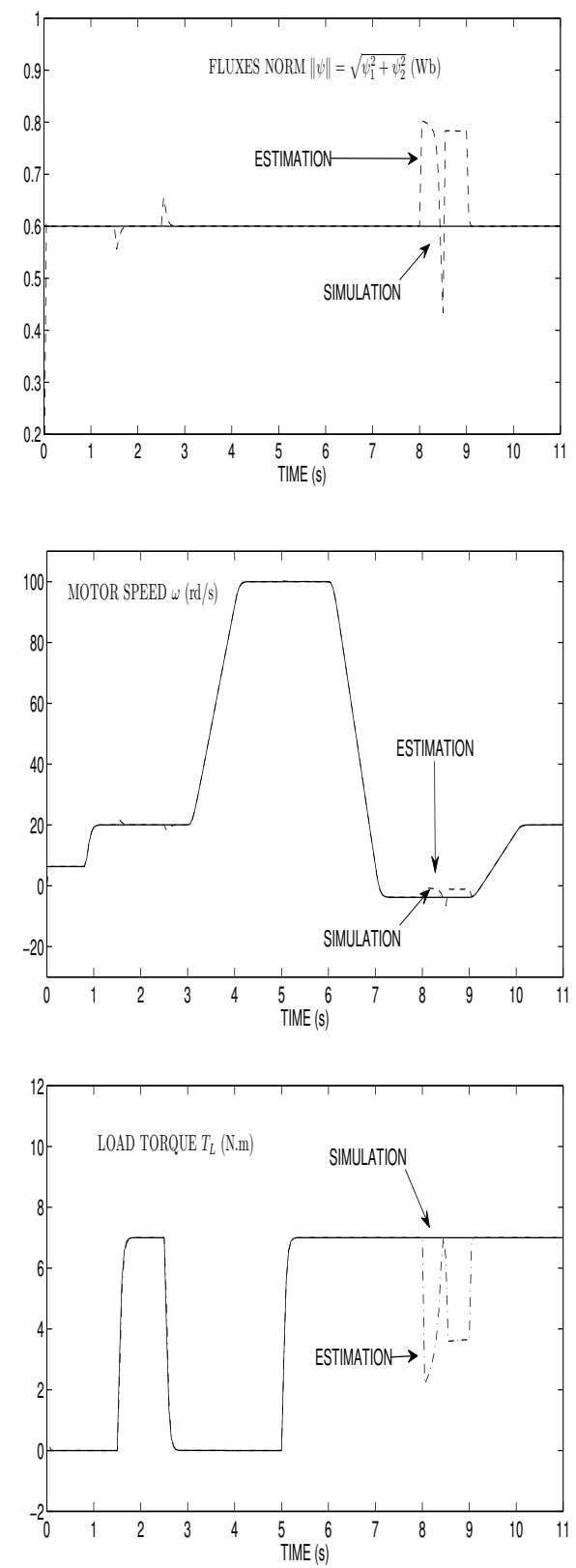

Fig. 1. Estimation results for $\|\psi\|, \omega$ and $T_{L}$

Recall that, the time evolution of the load torque as well as the moments when the IM model becomes unobservable are not known by the observer which still run with the same value of the tuning parameters: $\theta=100, k_{1}=k_{2}=3, k_{3}=1$. The value of the parameter $\delta$ has been fixed to $10^{-8}$.

\section{CONCLUSION}

In this paper, a simple observer is derived to estimate the full state of the IM from the current and voltage measurements. The simplicity of the proposed observer lies in the ease of its implementation and calibration. Indeed, the gain of the observer does not necessitate the resolution of any dynamical system and is explicitly given. The observer synthesis was preceded by an observability analysis that allowed to derive a sufficient condition ensuring the observability of the IM model. Simulation experiments around the unobservability neighborhood have been carried out and the obtained results clearly demonstrated the good ability of the proposed observer in providing accurate estimates of the missing states. The use of the proposed observer in sensorless feedback control scheme is under study and will be presented in forthcoming works.

\section{REFERENCES}

A. Benchaib, A. Rachid, E. Audrezet, and M. Tadjine. Real-time sliding-mode observer and control of an induction motor. IEEE Trans. Ind. Electron., 46(1):128-138, 1999.

K. Busawon, H. Yahoui, H. Hammouri, and G. Grellet. A nonlinear observer for induction motors. The European Physical Journal. Applied Physics, 15:181-188, 2001.

C. Darengosse, P. Chevrel, I. Souleiman Guelle, and S. Siala. A LMI-based observer for induction motor. In Proc. of ECC'99, Karlsruhe, Germany, 1999.

M. Farza, M. M'Saad, and L. Rossignol. Observer design for a class of MIMO nonlinear systems. Automatica, 40:135-143, 2004.

M. Ghanes and G. Zheng. On sensorless induction motor drives; sliding-mode observer and output feedback controller. IEEE Trans. on Industrial Electronics, 56:3404-3413, 2009.

M. Ghanes, J.P. Barbot, J. De leon, and A. Glumineau. A robust sensorless feedback controller of the induction motor drives: New design and experimental validation. Int. J. of Control, 83:484-497, 2010 .

J. Guzinski, H. Abu-Rub, M. Diguet, Z. Krzeminski, and A. Lewicki. Speed and load torque observer application in high-speed train electric drive. IEEE Trans. on Industrial Electronics, 57:565-574, 2010.

S. Hajji, M. Farza, M. M'Saad, and M. Kamoun. Observer-based output feedback controller for a class of nonlinear systems. In Proc. of the 17th IFAC World Congress, Seoul, Korea, 2008.

R. Hermann and A. Krener. Nonlinear controllability and observability. IEEE Transaction on Automatic Control, 22:728-740, 1977.

J. Holtz. Sensorless Control of AC Motor Drives, chapter Methods for speed sensorless control of AC drives. Piscataway, NJ, 1996.

S. Ibarra-Rojas, J. Moreno, and G. Espinosa-P'erez. Global observability analysis of sensorless induction motors. Automatica, 40: 1079-1085, 2004.

C. Lascu, I. Boldea, and F. Blaabjerg. A class of speed-sensorless sliding-mode observers for high performance induction motor drives. IEEE Trans. on Industrial Electronics, 56:3394-3403, 2009.

W. Leonard. Control of electrical drives. Springer-Verlag, 2001. 3rd edition.

D. Lubineau, J.M. Dion, L. Dugard, and D. Roye. Design of an advanced nonlinear controller for induction motor and experimental validation on an industrial benchmark. The European Physical Journal Applied Physics, 9:165-175, 2000.

Ph. Martin and P. Rouchon. Two simple flux observers for induction motors. International Journal of adaptive control and signal processing, 14(2/3):171-176, 2000.

R. Ortega, A. Loria, P. J. Nicklasson, and H. Sira-Ramirez. Passivitybased control of Euler-Lagrange systems: Mechanical, Electrical and Electromechanical Applications. Springer-Verlag, 1998. London.

L. Rossignol, M. Farza, and M. M'Saad. Nonlinear observation strategies for induction motors. In Proc. of the IEEE International Electric Machines and Drives Conference,IEMDC'03, pages 696-702, Madison, Wisconsin, 2003.

H. Tajima and Y. Hori. Speed-sensorless field-orientation control of the induction machine. IEEE Trans. Ind. Applicat, 29(1):175-180, 1993.

G.C. Verghese and S.R. Sanders. Observers for flux estimation in induction machines. IEEE Trans. on Automatic Control, 35(1): 85-94, 1988.

C. Canudas De Wit, A. Youssef, J.P. Barbot, Ph. Martin, and F. Maltrait. Observability conditions of induction motors at low frequencies. In Proc. 39th IEEE Conference on Decision and Control, Sidney, Australia, 2000. 